Meta

Journal des traducteurs

Translators' Journal

\title{
Les paradoxes de la créativité en traduction littéraire
}

\section{Lance Hewson}

Volume 62, numéro 3, décembre 2017

La traduction littéraire comme création

URI : https://id.erudit.org/iderudit/1043945ar

DOI : https://doi.org/10.7202/1043945ar

Aller au sommaire du numéro

\section{Éditeur(s)}

Les Presses de l’Université de Montréal

ISSN

0026-0452 (imprimé)

1492-1421 (numérique)

Découvrir la revue

Citer cet article

Hewson, L. (2017). Les paradoxes de la créativité en traduction littéraire. Meta, 62(3), 501-520. https://doi.org/10.7202/1043945ar

\section{Résumé de l'article}

Le présent article se donne pour objectif de proposer un cadre interprétatif qui permet de repérer des zones de créativité dans une traduction littéraire. Une partie introductive consacrée aux paradoxes qui émergent de l'association entre traduction et créativité est suivie d'une réflexion sur les limites des définitions usuelles de la créativité dans un contexte de traduction. Trois éléments sont critiqués : l'idée que seul un "problème " puisse déclencher le processus créatif, les insuffisances du critère de "nouveauté » et le flou qui entoure le rôle et les compétences de l'« expert » censé établir la manifestation de créativité. Un nouveau cadrage est proposé qui détaille les compétences de l'expert, l'importance du cadre interprétatif, les niveaux d'analyse et la démarche analytique. À ce dernier égard, l'accent est mis sur la distinction entre choix de traduction conscients et semi-automatiques, et sur la reconstitution des choix traductifs potentiels. Enfin, deux cas particuliers sont examinés : l'addition et la transformation. La dernière partie de l'article porte sur l'examen d'exemples tirés des versions croate et française d'un roman de T. Pynchon, et de deux traductions anglaises de Madame Bovary.
Ce document est protégé par la loi sur le droit d'auteur. L'utilisation des services d’Érudit (y compris la reproduction) est assujettie à sa politique d'utilisation que vous pouvez consulter en ligne.

https://apropos.erudit.org/fr/usagers/politique-dutilisation/ 


\title{
Les paradoxes de la créativité en traduction littéraire
}

\author{
LANCE HEWSON \\ Université de Genève, Suisse \\ lance.hewson@unige.ch
}

\begin{abstract}
RÉSUMÉ
Le présent article se donne pour objectif de proposer un cadre interprétatif qui permet de repérer des zones de créativité dans une traduction littéraire. Une partie introductive consacrée aux paradoxes qui émergent de l'association entre traduction et créativité est suivie d'une réflexion sur les limites des définitions usuelles de la créativité dans un contexte de traduction. Trois éléments sont critiqués: l'idée que seul un «problème» puisse déclencher le processus créatif, les insuffisances du critère de «nouveauté» et le flou qui entoure le rôle et les compétences de l'«expert» censé établir la manifestation de créativité. Un nouveau cadrage est proposé qui détaille les compétences de l'expert, l'importance du cadre interprétatif, les niveaux d'analyse et la démarche analytique. À ce dernier égard, l'accent est mis sur la distinction entre choix de traduction conscients et semi-automatiques, et sur la reconstitution des choix traductifs potentiels. Enfin, deux cas particuliers sont examinés: l'addition et la transformation. La dernière partie de l'article porte sur l'examen d'exemples tirés des versions croate et française d'un roman de T. Pynchon, et de deux traductions anglaises de Madame Bovary.
\end{abstract}

\section{ABSTRACT}

The aim of this article is to set out an interpretative framework enabling the researcher to identify instances of creativity in a literary translation. The introduction looks at how creativity and translation form a paradoxical association. Attention is then turned to the usual explanations that are given of creativity in the translational context. Three criteria are critically examined: that there must be a "problem" for creativity to come into play, that the result must be "new," and that an "expert" - a term generally left undefined must be involved in judging the product. A new framework is then set out, which looks at the expert's competences, the importance of the critical framework, the levels of analysis and the analytical steps to be followed. These steps include making a distinction between conscious and semi-automatic translational choices, and reconstructing the potential choices that the translator could have made. The particular issues associated with addition and transformation are also examined. The final part of the article looks at a small corpus of examples taken from the Croatian and French translations of one of Pynchon's novels, and two English translations of Madame Bovary.

\section{RESUMEN}

El presente artículo tiene como objetivo presentar un marco interpretativo que permita identificar zonas de creatividad en una traducción literaria. En la introducción se aborda la relación paradójica existente entre creatividad y traducción. Le sigue una reflexión sobre los límites de las definiciones más frecuentes de creatividad en un contexto de traducción. Se critican tres elementos: la idea de que solo un «problema» pueda desencadenar el proceso creativo, las insuficiencias del criterio de «novedad» y la falta de claridad en torno al papel y a las aptitudes del «experto» que debe decidir cuándo hay una expresión de creatividad. Se propone un nuevo enfoque en el que se detallan las aptitudes del experto, la importancia del marco interpretativo, los niveles de análisis y el método analítico. Acerca de este último punto, se hará hincapié en la distinción entre las opcio- 
nes de traducción conscientes y las semiautomáticas, y en la reconstitución de las opciones de traducción potenciales. Para terminar, se examinan dos casos particulares: la adición y la transformación. La última parte del artículo consiste en el análisis de ejemplos extraídos de las versiones croata y francesa de una novela de T. Pynchon, y de dos traducciones inglesas de Madame Bovary.

\section{MOTS CLÉS/KEYWORDS/PALABRAS CLAVE}

cadre interprétatif, démarche analytique, choix conscients, choix semi-automatiques, imprévisibilité

interpretative framework, analytical steps, conscious choices, semi-automatic choices, unpredictability

marco interpretativo, método analítico, opciones conscientes, opciones semiautomáticas, imprevisibilidad

\section{Introduction}

C’est une évidence: traduction ne rime pas avec créativité. Pourtant, cette évidence cache une réalité complexe, qui est celle du rôle que peut jouer la créativité en traduction littéraire. Pourquoi en traduction littéraire plutôt qu'en traduction tout court? La question mérite d'être posée, surtout lorsque l'on sait que la plupart des publications qui traitent de la problématique de la créativité en traduction se focalisent sur des textes pragmatiques. Or, la traduction littéraire a ses propres enjeux: le travail sur la forme et le fond que doit mener le traducteur littéraire exige des compétences de réécriture particulières; la créativité compte selon moi parmi les plus importantes. Pourtant, nous butons d'emblée sur une série de paradoxes, dont quatre me semblent particulièrement pertinents. Le premier concerne le statut de la créativité, car celle-ci est tout à la fois désirable et indésirable: désirable, parce qu'elle peut conduire à des solutions de traduction qui enthousiasment le chercheur-critique; indésirable, parce qu'elle peut aussi mener à des excès que celui-ci se doit de déplorer. Le deuxième paradoxe se fait jour lorsqu'on s'interroge sur la possibilité même de la créativité dans un contexte de traduction. En effet, toute désirable qu'elle soit, la créativité semble, à première vue, inconcevable, dans la mesure où l'on peut estimer qu'elle entre en contradiction avec la nature même de la traduction. La traduction, opération de réécriture à partir d'un texte existant, n'est pas directement assimilable à l'écriture, qui est le propre du texte original - écriture que l'on associe volontiers à la créativité. Le troisième paradoxe apparaît quand on examine le texte traduit, qui contient tout au plus des moments de créativité: on est effectivement loin du phénomène global que l'on associe à l'acte de création littéraire. Notons enfin que, pour le lecteur de la traduction, cette créativité éventuelle reste non seulement invisible, mais aussi impensable car, au moins depuis le géomètre de Montesquieu ${ }^{1}$, et surtout depuis l'avènement de la traduction automatique (Hewson 2016: 26), la traduction est généralement assimilée à une opération dénuée d'intérêt et, surtout, de créativité. Nous verrons, cependant, que nous sommes en présence de paradoxes révélateurs, qui permettent d'explorer la nature de ces «moments» de créativité dont peut témoigner un texte cible. Mon propos, en effet, n'est pas de contribuer aux écrits, déjà nombreux, qui portent sur la nature du processus créatif, mais uniquement d'explorer le produit issu de ce processus - produit qui, paradoxalement, devrait préoccuper le chercheur avant même que celui-ci n'entame une réflexion sur les étapes qui y mènent. Car, si 
on ne sait pas reconnaître les caractéristiques d'un produit (en partie) créatif, sur quelles bases peut-on explorer le processus qui a permis son émergence?

\section{Tentatives de définition et de cadrage}

Les traductologues semblent unanimes pour considérer que, bien qu'il existe de nombreuses définitions de la créativité, toute tentative de trouver une seule et même définition applicable dans tous les cas de figure est vouée à l'échec. Bayer-Hohenwarter, par exemple, déclare:

(t)he various conceptions and definitions of creativity that can be found in the psychological literature on creativity research and in translation research are so multi-faceted that any attempt to describe its complexity in an exhaustive manner and provide the definition of translational creativity is bound to fail. (Bayer-Hohenwarter 2011: 664, les italiques sont de l'auteur)

Par conséquent, on se rabat sur la caractérisation des étapes censées se succéder tout au long de la «performance» créative (processus et produit). En témoignent les nombreux travaux de Kussmaul dans le domaine, notamment la manière dont il aborde la créativité dans son ouvrage Kreatives Übersetzen:

Eine kreative Leistung entsteht aufgrund einer Problemerkenntnis und stellt etwas Neues dar, das zu einer bestimmten Zeit in einer Kultur von Experten als angemessen akzeptiert wird. (Kussmaul 2000a: 20, les italiques sont de l'auteur).

Le processus créatif s'articulerait donc en trois étapes. Il doit y avoir, tout d'abord, prise de conscience d'un problème de traduction qui, lors de sa résolution, donne lieu à quelque chose de nouveau; ce quelque chose doit être validé par des experts. À première vue, ce découpage, qui emporte l'adhésion d'autres traductologues comme Bastin (2003), Fontanet (2005) ou Dancette et al. (2007), semble satisfaisant, car les deux premiers éléments correspondent effectivement au vécu du traducteur. Celui-ci se heurte constamment à divers types de problèmes; il peut avoir l'impression que, parmi les solutions trouvées, certaines sont vraiment "nouvelles», et sont, par conséquent, créatives. Par ailleurs, puisqu'on veut être en mesure de juger le travail du traducteur, il est normal que sa "performance» soit validée par une instance extérieure. En réalité, cependant, les choses ne sont pas aussi simples. En effet, chacune des étapes comporte son lot de problèmes; prises ensemble, elles ne constituent pas une base satisfaisante pour examiner le phénomène de la créativité. C’est ce que je vais essayer de démontrer avant de proposer un changement de paradigme.

\subsection{La problématique du «problème»}

Beaucoup de chercheurs s'accordent à dire que l'identification d'un problème de traduction produit l'étincelle qui met en branle le processus créatif. Un problème, écrit Fontanet, correspond à

toute situation dans laquelle le traducteur se trouve dans l'incapacité de produire spontanément un équivalent du texte de départ en langue d'arrivée, dans la mesure où il prend conscience de cette incapacité et y remédie en passant par un processus de recherche de solution. (Fontanet 2005: 433) 
Cette vision rappelle les travaux d'autres traductologues. On peut, à ce propos, citer l'article de Balacescu et Stefanink (2005), qui est entièrement axé sur la résolution de problèmes de traduction, ou l'ouvrage de Kussmaul (2000a), qui met en exergue le lien étroit entre les efforts consentis pour résoudre des problèmes et la créativité. Cependant, il me semble dangereux de faire de cette "problématique du problème» un paramètre incontournable, dont la présence permettrait de prévoir un comportement traductif axé sur la créativité, et ce, pour deux raisons.

La première raison concerne le lien supposé entre l'existence d'un problème, quel qu'il soit, et l'adoption d'une démarche créative par le traducteur. En effet, chaque traducteur a sa propre manière de procéder, ce qui laisse supposer qu'en réaction à un seul et même problème, certains adopteraient une démarche créative tandis que d'autres pourraient se contenter d'une solution minimaliste, voire d'un refus de traduire (Hewson 2004). Chaque commande de traduction est sui generis, exécutée dans des circonstances spécifiques pour le compte d'un commanditaire ayant ses propres exigences. Enfin, quelle unité convient-il d'analyser, sachant que même si on examine une proposition courte, plusieurs problèmes de nature différente (relevant, par exemple, du lexique, de la syntaxe ou du style) peuvent se conjuguer? Rien ne permet d'affirmer, à ce stade, que l'existence d'un problème va nécessairement déclencher une démarche créative.

La seconde raison que je souhaite avancer est très simple: on doit admettre non seulement que l'existence d'un problème peut ne pas amorcer une démarche créative, mais aussi que cette même démarche peut survenir en l'absence de problèmes ou de difficultés. Nous sommes ici au cœur même de la problématique de la traduction littéraire, dont l'une des préoccupations principales est la création d'un style adéquat en langue cible. Tout traducteur littéraire sait qu'une section de texte source d'apparence simple peut résister à une traduction satisfaisante en langue cible. Par ailleurs, il serait incorrect d'imaginer que le traducteur ne déploie sa créativité que lors de la première phase de son travail, celle qui débouche sur la première version complète du texte cible. On peut, en effet, estimer que pendant la phase finale de révision de la traduction, qui survient après la résolution de toutes les difficultés (et des nondifficultés...) rencontrées, le traducteur s'autorise à procéder à une réécriture partielle du texte qu'il a produit en langue cible, en introduisant de nouvelles touches dont certaines sont certainement créatives.

Précisons toutefois qu'il n'est nullement question d'exclure la problématique du "problème», mais uniquement d'y voir un indicateur d'une démarche potentielle en matière de créativité.

\section{2. À la recherche de la solution "nouvelle»}

Ce deuxième critère est celui qui s'impose sans doute spontanément à l'esprit lorsqu'on examine la problématique de la créativité en général. Il est mis en exergue dans la définition de la créativité donnée dans le Trésor de la langue française informatisé, citée par Fontanet (2005: 433): «Capacité de découvrir une solution nouvelle, originale, à un problème donné » ${ }^{2}$. D'autres chercheurs, à l'instar de Balacescu et Stefanink (2003a), s'appuient sur le concept de nouveauté. Cependant, il ne faut jamais perdre de vue que nous sommes dans un contexte de traduction, qui présuppose un processus dont le résultat, le texte cible - tout en étant nécessairement différent du 
texte source -, n'est pas vraiment nouveau. Cette constatation explique la démarche de certains chercheurs consistant à suggérer d'autres termes qui permettraient d'exprimer l'idée de nouveauté tout en restant dans un cadre traductologique. C'est dans ce sens que va Kussmaul lorsqu'il propose une palette complète de critères:

[t] he creative product must be both novel and useful, it must contain an element of surprise, but must also fulfil certain needs, it must be singular or at least unusual, but at the same time must fit in with reality. (Kussmaul 1991: 92)

Le premier terme, "novel», est un choix intéressant, car il ne correspond ni au terme choisi dans le premier extrait de Kussmaul cité plus haut ("etwas Neues») ni à l'adjectif français nouveau. En effet, il introduit l'idée que la solution doit être intéressante et/ou inhabituelle ${ }^{3}$, idée renforcée plus tard dans la citation par les termes «singular» et « unusual». Le deuxième critère énoncé, « useful», vise à écarter toute solution dont la pertinence serait discutable. Le troisième, "an element of surprise», prévoit - assez curieusement - la réaction d'un lecteur qui, parce qu'il doit nécessairement avoir accès à l'original, ne peut en fait être que le traducteur lui-même, qui valide son propre choix, ou le chercheur-critique qui, plus tard, compare les textes source et cible. Enfin, Kussmaul revient sur la nécessaire pertinence du choix en ajoutant un autre critère ("fit in with reality»), qui, tout comme "useful», vise à écarter les propositions de traduction qui ne seraient pas conformes, mais dont le critère de conformité reste on ne peut plus flou («reality»). Notons enfin que novel est repris par d'autres traductologues s'exprimant en anglais (e.g. Bayen-Hohenwarter 2011:664), et que ce terme correspond à la paire novateur + inattendu, utilisée dans la définition de créativité proposée dans le Dictionnaire des sciences cognitives et reprise par Dancette et al. (2007: 109) ${ }^{4}$.

Que conclure de ces tentatives de cerner les caractéristiques d'une traduction créative? Il est clair que la multiplication des termes employés ne permet ni de définir son fondement d'un point de vue empirique ni de reconnaître sans hésitation la nature du produit final. La confusion qui règne révèle par ailleurs une théorisation excessive, sans doute héritée des disciplines dont les traductologues s'inspirent, bien éloignée du cadre effectif qui accompagne tout acte de traduction. J'essaierai dans ma troisième partie de proposer une autre approche.

\subsection{La validation par des "experts"}

Ce critère, annoncé par Kussmaul et repris par d'autres traductologues (Balacescu et Stefanink 2003a) semble aller de soi: sans mécanisme de validation, il serait impossible de faire le départ entre un choix traductif jugé créatif et pertinent, et un choix à première vue créatif, mais écarté dans la mesure où il est jugé non pertinent ou aberrant. Force est cependant de constater que les traductologues restent muets lorsqu'il s'agit de préciser l'identité d'un tel expert, les compétences requises ou les critères qu'il convient de mobiliser. C'est le cas de Kussmaul, qui, dans un article paru en 2000 qui vise à créer une typologie des traductions créatives, énonce clairement que sa recherche s'inspire de «bonnes traductions». Il écrit notamment: «I am specifically studying good translations. They can be taken as models of how to translate successfully, especially if the cognitive categories behind them can be revealed» (Kussmaul 2000b: 118). Son argumentation s'appuie de fait sur plusieurs exemples, 
notamment les traductions allemande et anglaise des noms propres de la bande dessinée Astérix. Or, s'il est vrai que des traductologues spécialisés dans ce sousdomaine louent en général la qualité de ces traductions (Delesse et Richet 2009), il me semble dangereux d'élaborer une problématique sans aucune discussion approfondie des critères utilisés et, en particulier, sans aborder l'épineuse question de la «bonne» traduction ${ }^{5}$.

Ainsi, les trois étapes généralement retenues dans les discussions portant sur la créativité s'avèrent problématiques. Je tâcherai, dans la section qui suit, de proposer un nouveau cadrage qui s'appuie sur des critères clairs et exploitables.

\section{Vers un nouveau cadrage de la créativité en traduction littéraire}

Il importe, au début de cette section, de rappeler que l'objet de ma démarche est de poser un cadre permettant de se prononcer sur la créativité à l'œuvre dans une traduction littéraire. Je conçois celui-ci comme une étape préalable, destinée, dans un second temps, à alimenter la recherche sur les processus créatifs engagés dans l'acte traductif. Je n'entends cependant pas minorer les efforts des chercheurs qui se sont déjà penchés sur ces processus, car ceux-ci donnent des résultats probants. Citons à titre d'exemple la contribution de Dancette et al. (2007), qui examinent les résultats d'études empiriques en distinguant la compréhension créative et la réécriture créative, ou celle de Bayer-Hohenwarter (2011), qui introduit le concept de «déplacements créatifs» ("creative shifts») pour rendre compte des processus qui permettent au traducteur de s'éloigner des structures du texte source. Un regard plus global sur le déroulement des processus est proposé par Kussmaul (1991), qui emprunte à la recherche en psychologie un modèle en quatre phases, allant de la préparation à l'évaluation, en passant par l'incubation et l'illumination. Enfin, d'autres chercheurs, comme Balacescu et Stefanink (2003a; 2005), se tournent vers la linguistique cognitive et mettent à contribution les recherches des grands noms du domaine, en l'occurrence E. Rosch, R. W. Langacker, C. J. Fillmore et R. C. Schank.

Le cadrage que je propose a pour vocation de permettre une évaluation du travail du traducteur dans l'optique de la créativité. Il établit tout d'abord la nécessité d'une «instance validante», appelée à se prononcer sur la créativité (ou l'absence de créativité) manifestée dans un texte cible (sous-section 3.1.). Je pose qu'un critère essentiel pour le bon déroulement du travail d'analyse est la mise en place d'un cadre interprétatif permettant une analyse à deux niveaux distincts (3.2.). L'addition et la transformation, phénomènes qui se manifestent souvent dans le texte cible, mais dont le rapport avec la créativité n'est pas évident, font l'objet d'une analyse complémentaire (3.3.). Enfin, un bilan (3.4.) permet de tirer quelques conclusions générales et d'amener à l'illustration empirique qui occupe la quatrième section de cet article.

\subsection{L'expert, ou l'instance validante}

On se souviendra que l'une des objections formulées à l'égard de la notion de validation, employée par Kussmaul dans sa définition de la "performance» créative, concerne l'expert appelé à se prononcer sur les caractéristiques du texte cible. Si le chercheur allemand a raison de considérer l'avis d'un expert comme incontournable, encore faut-il savoir qui peut remplir ce rôle d'«instance validante». Avant même 
d'examiner comment cette personne peut entrer en matière, il convient de dresser son portrait-robot. L'expert doit être en mesure d'intervenir à trois niveaux. Le premier niveau est le niveau traductologique proprement dit, qui implique une connaissance de la spécificité de la traduction et du discours académique qui l'accompagne. Cette connaissance peut certes sembler évidente, mais il ne faut pas perdre de vue que de nombreux spécialistes travaillant dans des domaines connexes évoquent volontiers la traduction et ses défis sans être nécessairement au courant des problématiques développées en traductologie ${ }^{6}$. Le deuxième niveau est spécifiquement lié au type de traduction envisagé ici, c'est-à-dire la traduction littéraire. Nous verrons que les choix traductifs influent directement sur les pistes interprétatives que peut suivre le lecteur en langue cible. Être capable de dégager ces pistes implique une capacité d'analyse critique et littéraire. Enfin, une mise en perspective des choix traductifs repose sur la capacité d'envisager des variantes paraphrastiques en langue cible: notre traductologue-critique est également traducteur.

\subsection{Le cadre interprétatif et les niveaux d'analyse}

Il serait vain de procéder à la validation de choix de traduction en dehors de tout cadre interprétatif établi en amont. Comme dans le domaine de la critique des traductions (Hewson 2011), le cadre interprétatif permet d'asseoir des prises de position argumentées; il constitue le fondement sur lequel s'appuient les arguments avancés par l'expert lorsque celui-ci se prononce sur des choix traductifs potentiellement créatifs. Il invite à un va-et-vient entre le niveau macrostructurel, où se dégagent les pistes de lecture jugées fondamentales, et le niveau microstructurel, examiné dans le cadre d'un choix traductif particulier. Or, il est intéressant de constater que la plupart des spécialistes semblent privilégier une analyse au seul niveau microstructurel. Dans cette optique-là, on se contente de concentrer l'attention sur un petit nombre d'éléments, tantôt au niveau du mot ou du groupe de mots, tantôt au niveau de la proposition ou de la phrase. C'est la démarche qu'adopte Kussmaul dans son ouvrage Kreatives Übersetzen (2000a: 43), et que préconisent également Balacescu et Stefanink (2003b: 138). Comme je le note dans une autre publication (Hewson 2006: 57-59), les analyses proposées dans les deux articles en question portent sur un seul et même exemple de difficulté de traduction. S'agissant d'une phrase tirée d'un article du magazine américain Newsweek, on pourrait sans doute estimer que ce regard microstructurel, qui ne dépasse pas la phrase en question, est suffisant. Le texte littéraire, en revanche, requiert une double vision, rendue possible par l'élaboration du cadre interprétatif. Dans cette optique, tout choix microstructurel est validé dans le cadre de son contexte étroit, puis considéré dans la perspective macrostructurelle établie en amont. On peut ainsi se prononcer sur la cohérence globale des choix et rester vigilant relativement à certaines options qui, à première vue, sont créatives, mais qui, en réalité, sont aberrantes. En effet, certains traducteurs cèdent à la tentation de se laisser inspirer par leur propre talent d'écrivain, produisant ce que Berman (1999: 40) appelait le dépassement, ce moment où, au lieu d'être au service de son auteur, le traducteur se permet de se faire plaisir et de laisser parler sa propre voix. C'est le phénomène de traduction ontologique que je décris dans d'autres publications (2004; 2011), où le traducteur privilégie l'écriture au détriment de la réécriture: son objet (avoué ou inconscient) est d'"exister» en tant qu'écrivain. Précisons, enfin, que le 
cadre interprétatif est l'endroit où s'exprime le point de vue, forcément subjectif, de l'expert. Son intégration dans le processus de validation garantit la visibilité des critères appliqués.

\subsection{La démarche analytique}

Beaucoup de chercheurs, comme je l'ai déjà fait remarquer, s’intéressent aux textes pragmatiques, et souvent à des textes courts. Si on peut raisonnablement entreprendre d'examiner un texte journalistique du début à la fin, le texte littéraire se prête rarement à une analyse de type exhaustif. On peut, certes, analyser une nouvelle ou un poème dans son ensemble (c'est ce que fait Koster (2000) dans son ouvrage sur la critique des traductions); cependant, une pièce de théâtre ou un roman exige un découpage préalable. Par conséquent, on doit prévoir un mécanisme qui permet de sélectionner des passages potentiellement intéressants (3.3.1.). On s'intéressera ensuite à la mise en perspective des choix du traducteur, en distinguant entre ceux qui sont clairement le résultat d'une démarche consciente, et ceux qui relèvent d'une pratique semi-automatique (3.3.2.). Par la suite, dans la même sous-section, j'écarterai l'idée qu'il peut y avoir des contraintes de traduction absolues. Enfin, nous verrons qu'il est très utile, lors de l'analyse du travail du traducteur, de reconstituer les choix traductifs potentiels, autrement dit ceux auxquels le traducteur a renoncé (3.3.3.).

\subsubsection{Le choix des passages}

La section consacrée à la "problématique du problème» a permis de comprendre l'orientation des chercheurs qui, lorsqu'ils définissent leur objet d'analyse, opèrent une distinction entre les unités dont la traduction semble poser de sérieux problèmes et celles qui se prêteraient à des solutions de routine. Bayer-Hohenwarter explique cette approche comme suit:

[f]or each experimental text, a number of units of analysis were defined. The selection criterion for those units of analysis was that they should be potentially promising and highly relevant for the research questions to be answered. In the case of a study on the development of translational creativity, such promising units of analysis bore either a high creativity potential or a low creativity potential (= a high routine potential). Units with a high creativity potential [...] are defined as problematic units that are deemed to require high problem-solving capacity. Units with a high routine potential [...] were such that they were regarded as fairly unproblematic, at least for experienced translators. For each experimental text, 2 creativity units and 2 routine units were selected. Even if it is acknowledged that the attribution of the label "creative" versus "routine" is subjective to a certain extent, this distinction proved generally consensual and useful in a pilot study [...]. (Bayer-Hohenwarter 2011: 671-672)

Dans le cadre de la traduction littéraire, cette manière de procéder s'avère utile jusqu'à un certain point, mais doit être modifiée pour prendre en compte les spécificités de ce type de traduction. Examinons d'abord les avantages de cette approche. On peut, en effet, estimer que la présence dans le texte source de certains éléments peut conduire à une démarche créative. Ces éléments sont de deux types. On peut, d'une part, relever la présence d'éléments dont on sait, pour la paire de langues en question, que le passage vers l'autre langue peut s'avérer problématique. Citons à titre d'exemple pour le couple français-anglais la traduction du pronom français on, ou celle de la 
préposition dès, dont un exemple de traduction créative est donné ci-après (4.2.). On peut aussi examiner la manière dont le texte source est ancré dans sa propre culture, en repérant tout ce qui est culturellement spécifique, comme les realia (Florin 1993). On sait que la transposition dans l'autre langue du culturellement spécifique n'est pas sans embûches. Une lecture attentive du texte source permet de cerner d'autres difficultés qui ne seraient pas généralisables, mais qui, manifestement, exigent un travail de réflexion de la part du traducteur. Les acronymes présents dans un roman de Pynchon, analysé au paragraphe 4.1., en constituent un exemple parlant. Cependant, cette approche montre rapidement ses limites, car, comme je l'ai indiqué ci-dessus, le traducteur littéraire est susceptible d'adopter une démarche créative pour des raisons qui, à première vue tout du moins, échappent à l'expert lorsque celui-ci fait ses repérages en amont. Le traducteur peut décider, par exemple, que la traduction standard d'une phrase, bien que correcte, ne rend pas l'effet de style recherché. Il peut constater que ses tentatives de traduire les diverses « voix» qui traversent le texte source exigent un effort particulier qui va au-delà du souci de "reproduire» celles-ci; il cherche donc à créer des voix qui obéissent à une logique "similaire», tout en respectant cette logique du début jusqu’à la fin de la traduction. Afin de débusquer les plages de texte susceptibles de déclencher le processus créatif, et sachant que celles-ci ne sont pas nécessairement repérées en amont, il convient de procéder à une comparaison des textes source et cible, en vue de révéler des choix traductifs insolites qui auraient échappé à un tri basé sur les critères du seul texte source. Cette démarche m’a permis de découvrir un exemple que j'exploite dans un autre article (Hewson 2014), où je commente une traduction anglaise du français tisanes, terme qui, a priori, ne présente aucune difficulté de traduction. Or, dans sa traduction de Madame Bovary, E. MarxAveling ([1886] 2006: 430) opte pour un mot inattendu, à savoir gruel ${ }^{7}$.

En conclusion, il convient de procéder en deux temps. Un repérage en amont permet d'identifier des passages potentiellement intéressants, correspondant au potentiel créatif mis en exergue par Bayer-Hohenwarter. Il est également important de compléter ses premières observations par des sondages pratiqués à divers endroits, choisis de manière aléatoire, dont l'objectif est d'offrir une vision plus complète de la démarche du traducteur. Il importe désormais d'envisager une méthodologie qui permettra d'atteindre cet objectif. Pour ce faire, nous devons tout d'abord explorer la manière dont le traducteur est susceptible de procéder.

\subsubsection{La distinction entre choix conscients et semi-automatiques}

Beaucoup de traductologues s'accordent à dire que le travail du traducteur oscille constamment entre deux démarches, l'une pleinement consciente et l'autre semiautomatique. On peut, à ce titre, citer les travaux de Levý, qui va jusqu'à parler de traducteurs «mécaniques» (1963/1983/2011 : 34), l'article de Balacescu et Stefanink (2003b : 131), qui parle de "préformés linguistiques situationnels, marqués culturellement, que le bon traducteur bilingue et bi-culturel doit avoir à sa disposition et placer de façon quasi automatique dans le discours de la langue cible», ou l'ouvrage d'Englund Dimitrova (2005: 26), qui oppose «automatic processing» et «problemsolving». Il est utile, dans ce contexte, de revenir à l'article de Fontanet, déjà cité. S'appuyant sur la citation du Trésor de la langue française informatisé donnée sous 2.2., elle précise ce qu'elle entend par la notion de créativité: 
[t]elle que je l'entends dans le contexte de la traduction, cette notion implique que le traducteur trouve une solution par ses propres ressources et non pas à la suite d'une recherche documentaire ou terminologique. À cette notion s'oppose celle d'automatisme, de réflexe et de mise à profit de l'expérience acquise [...]. (Fontanet 2005: 433)

Cette formulation, qui dit clairement que certains processus ne relèvent pas d'une démarche créative, mérite un développement. Il est logique, tout d’abord, de préciser que la recherche documentaire ou terminologique en est exclue d'office, car, dans ce cas de figure, le traducteur ne fait que valider un choix existant et disponible. On ne peut qu'abonder dans le sens de la traductologue genevoise lorsqu'elle avance l'idée que la mise à profit de l'expérience est, elle aussi, à écarter. Il convient, en revanche, de s'interroger sur le statut à donner aux «automatismes» et aux «réflexes». Il me semble que nous sommes ici partagés entre, d'une part, le vécu linguistique et professionnel du traducteur en tant qu'individu et, d'autre part, l'organisation collective du discours (Guillemin-Flescher 1986). Le vécu du traducteur fournit des solutions déjà testées, qui sont intégrées dans l'exercice de son travail. Notons que celles-ci sont mobilisées en fonction des contenus du texte source, qui est en général rédigé dans une langue «étrangère» pour le traducteur. Les réflexes, en revanche, font partie de la langue maternelle qui, selon la célèbre formule de Gadamer, «nous parle» ${ }^{8}$. C'est à ce niveau que l'on peut faire une distinction entre le traducteur «alimentaire» et celui qui ambitionne de puiser dans les ressources de sa langue dans une optique de créativité. Le premier ne réfléchit pas quand il constate qu'il peut mécaniquement retranscrire des solutions déjà testées: son comportement est (semi-) automatique. Le second, par contre, est sensible aux «pressions» de la langue, tout en soupçonnant qu'à tout instant, un écart doit être possible. Non pas qu'il souhaite bouleverser la grammaire de sa langue: sa démarche consiste à exploiter sa richesse paraphrastique.

Il importe, à ce stade, d'apporter une précision concernant la liberté que peut exercer le traducteur. Cette liberté est, certes, relative, mais elle est toujours présente, en dépit de la double contrainte qui semble peser sur tout acte traductif. En quoi consiste la double contrainte? Nous avons, d'une part, un texte source dont les contenus sont à transmettre en langue cible, en suivant l'interprétation privilégiée par le traducteur. À cette première contrainte s'ajoutent, d'autre part, les normes rédactionnelles de la langue cible. Prises ensemble, ces deux contraintes font dire à certains traductologues que la marge de manœuvre du traducteur est limitée, et qu'il existerait, par ailleurs, des contraintes absolues auxquelles le traducteur ne peut échapper. Cependant, comme je l'explique dans un récent article (Hewson 2017), l'idée de contraintes absolues relève d'une vision prescriptive de la traduction, héritée d'une tradition qui privilégie une traduction de type linéaire ou mot à mot. Une approche descriptive, en revanche, permet de constater deux choses: certaines traductions ne sont pas intégrales (Hewson 2004), et, surtout, certains traducteurs proposent des choix de traduction inattendus ou surprenants. Il s'ensuit qu'une contrainte à première vue «absolue» - une interprétation qui s'imposerait à tout lecteur du texte source ou une formulation qui serait imposée par la grammaire de la langue cible - peut se transformer en opportunité: celle d'adopter une démarche créative. L'exemple tiré de Madame Bovary en anglais que j'ai cité en 3.3.1. est intéressant à ce propos, surtout lorsqu'on sait qu'à l'époque les traductions standard de tisane, herb tea et tisane, étaient utilisées couramment depuis, respectivement, le XVIII et le $\mathrm{XV}^{\mathrm{e}}$ siècle... ${ }^{9}$. On comprend ainsi que le choix de gruel est d'autant plus intéressant à analyser. 


\subsubsection{La reconstitution des choix traductifs potentiels}

L'optique que je viens d'esquisser permet d'envisager une méthodologie fondée sur la reconstitution des choix traductifs potentiels. En effet, si on se limite au texte cible tel qu'il a été publié, on écarte de l'analyse le potentiel traductif de la langue cible. Aussi convient-il de recréer l'acte traductif, avec toutes les précautions d'usage, et d'explorer le potentiel ainsi dégagé. En examinant le potentiel traductif d'un texte source, l'expert se donne les moyens de prendre en considération les choix que le traducteur a décidé d'écarter et, partant, de mieux jauger la solution effectivement choisie. Il est possible d'évaluer le degré de prévisibilité du texte cible, que l'on pourrait placer sur une échelle allant des solutions qui s'imposent toutes seules, aux vraies trouvailles. Il convient, cependant, de noter que ce travail a posteriori sur les processus a ses limites. À titre d'exemple, on peut examiner le statut qu'il convient de donner à la traduction dite littérale. À première vue, celle-ci semble correspondre à un choix semi-automatique: Newmark (1988), par exemple, voit dans ce type de traduction le modèle que l'on applique par défaut, à condition que le résultat soit acceptable. C'est sans doute ce modèle qui fait dire à des chercheurs tels que BayerHohenwarter (2011) que la créativité est à chercher dès l'instant où l'on constate que le traducteur opte pour une solution non littérale. Toutefois, en réalité, les processus sont beaucoup plus complexes. On peut estimer, par exemple, que c'est le réflexe du traducteur anglophone qui le conduit vers une reformulation non littérale lorsque celui-ci transforme des noms verbaux en verbes. La démarche consciente dans cet exemple consisterait à ne pas choisir la forme dictée par le réflexe du traducteur (c'està-dire une recatégorisation) et, après réflexion, à privilégier la traduction littérale. Il est clair que, dans des circonstances analogues, les protocoles de verbalisation, qui fournissent un cadre permettant au traducteur de s'exprimer sur les étapes de son raisonnement (Englund-Dimitrova 2005), peuvent éclairer le traductologue dans sa démarche de classification.

On comprend mieux, à la lumière de ces considérations, que le rôle que peut jouer la reconstitution des choix traductifs potentiels est circonscrit par la subjectivité de l'expert. Nous avons, in fine, deux subjectivités en jeu: celle du traducteur, dont les choix traductifs oscillent entre création et reproduction, et celle de l'expert, dont le vécu est forcément différent. Cependant, cette subjectivité est la condition sine qua non de l'exercice envisagé: elle doit être non seulement assumée, mais aussi affichée dans le cadre d'une présentation argumentée.

\subsection{Deux cas particuliers: l'addition et la transformation}

Ici, je souhaite examiner deux phénomènes particuliers que le lecteur-critique peut repérer lorsqu'il examine un texte cible. Laddition et la transformation ont un point en commun: elles conduisent à une modification de l'interprétation du texte cible (par rapport, bien entendu, à celle du texte source). L'idée de modification rappelle celle de nouveauté évoquée en 2.2. Par conséquent, il est intéressant de voir dans quelle mesure une modification d'interprétation peut relever d'une démarche créative. Prenons d'abord le cas de l'addition, qui, selon la définition que je donne dans l'article déjà cité, 
s'observe dès lors qu'un passage du texte cible comporte une information (i) qui n'est pas formulée dans le passage correspondant du texte source et (ii) dont il est considéré que le lecteur du texte source ne peut pas l'inférer. Cette opération modifie l'interprétation du texte. (Hewson 2017: 220)

Nous avons donc affaire à un élément inattendu, forcément «nouveau », dont la présence n'est pas, au simple regard du texte source, prévisible. Puisqu'il serait irresponsable de valider d'office toute addition repérée dans une traduction, le rôle de l'expert, qui se prononce en fonction de son cadre interprétatif, est primordial. Dans la présentation que je donne dans Hewson 2012 des traductions croate et française de No Man's Land du regretté Harold Pinter, je cite un exemple d'addition où le traducteur cherche manifestement à combler une lacune culturelle chez les spectateurs francophones:

(1) Spooner: I often hang about Hampstead Heath myself, expecting nothing.

(Pinter 1975: $18^{10}$ )

SPOONER: Je traînaille souvent par ici, entre les demeures policées et les frondaisons $\mathrm{du}$ parc, mais je n'espère rien.

(Pinter 1979: 13, adaptation de Kahane ${ }^{11}$ )

On peut supposer que le dramaturge compte sur les connaissances culturelles des spectateurs britanniques, qui n'auront pas de difficultés à visualiser ce quartier cossu de Londres avec son parc. Les additions de Kahane sont intéressantes de deux points de vue. Elles apportent une petite touche visuelle permettant d'imaginer le contexte auquel Spooner fait référence; elles contribuent par ailleurs à la manière alambiquée de s'exprimer qu'affectionne ce personnage. En bref, les additions examinées constituent un apport qui, tout en enrichissant le texte, sont en adéquation avec les pistes interprétatives que l'on peut dégager.

Il est intéressant d'examiner un autre exemple d'addition, dont les conséquences sont moins heureuses. Il s'agit de la traduction anglaise d'un roman de Simenon, traduction qui, comme je le démontre dans Hewson 2017, modifie passablement la voix narrative et la vision qu'a le lecteur du personnage principal. Dans ce passage, Maigret se trouve dans le bureau du juge d'instruction:

(2) Deux ou trois fois, la veille, le juge Coméliau avait téléphoné à son bureau.

(Simenon 1955/2012: $77^{12}$ )

By the time he had got back to his office the previous evening, there were several messages on his pad requesting him to ring Judge Coméliau.

(Ellenbogen 1967/1971:22013)

Outre les modifications que subit la voix narrative, on aura observé que les additions (le lecteur de l'original ne sait pas si Maigret est effectivement retourné à son bureau ni comment il a été informé des appels du juge; enfin, il n’est nullement précisé qu'il possède un carnet...) créent une autre image de la manière dont Maigret mène ses enquêtes. Ces modifications nuisent à l'interprétation du livre; par conséquent, dans l'optique qui est la mienne, ces choix traductifs ne relèvent pas d'une démarche créative.

On aura compris que, en matière de créativité, le cadre interprétatif joue un rôle primordial. Son importance transparaît également dans l'exemple de transformation 
qui suit, tiré de l'une des traductions françaises d'Emma (Austen [1816] [1966] 1970). Le terme transformation indique que le traducteur a privilégié une piste interprétative qui n'est pas prévisible eu égard au texte source. Autrement dit, le sens du texte cible est manifestement différent. Dans le quatrième chapitre de mon ouvrage sur la critique des traductions (2011), je mets en avant l'importance du contexte social dans les romans de la romancière anglaise. Puisque celui-ci constitue une clef de lecture incontournable, il est souhaitable que le lecteur du texte cible puisse avoir accès aux éléments essentiels de ce contexte. Or, on constate que celui-ci est malmené dans les traductions que j'examine. Nordon, par exemple, introduit une transformation dans la scène de demande en mariage, qui réunit Emma Woodhouse et Mr. Elton. Il s'agit d'un détail de comportement:

(3) ...but scarcely had she begun, scarcely had they passed the sweep-gate and joined the other carriage, than she found her subject cut up - her hand seized - her attention demanded, and Mr Elton actually making violent love to her.

(Austen 1816/1966/1970: 149 ${ }^{14}$ )

(a) Mais à peine avait-elle eu le temps de commencer, et à peine avaient-ils franchi la grille de la propriété pour rejoindre la première voiture, qu'il l'interrompit, lui prit la main, approcha son visage, et entreprit brusquement de lui déclarer son amour.

(Austen 1996: 138, traduction de Nordon ${ }^{15}$ )

Outre l'importante modification de la transitivité (Emma, en anglais, est la victime qui subit le comportement du vicaire), on note que le traducteur a gommé l'idée exprimée par «her attention demanded", tout en imaginant que Mr. Elton esquisse un mouvement vers l'avant («approcha son visage»), constituant ainsi un envahissement de l'espace privé de la jeune fille. Un tel comportement serait inconcevable dans ce contexte de début du $\mathrm{XIX}^{\mathrm{e}}$ siècle. Cette constatation nous amène à conclure que, loin d'être validable en tant qu'exemple de créativité, il s'agit d'un choix traductif qui nuit à l'interprétation macrostructurelle du roman.

Cette conclusion concernant la transformation est, somme toute, assez logique. Même si le traducteur peut, lui, estimer que sa démarche est créative, il incombe à l'expert de mesurer l'impact macrostructurel d'un tel choix. Le cas de l'addition est plus complexe: les deux exemples ci-dessus illustrent deux résultats différents, positif dans un cas et négatif dans l'autre. De toute manière, il est clair qu'il faut examiner de près de tels moments d'inspiration, avant de pouvoir arriver à une conclusion en matière de créativité.

\subsection{Bilan}

Le cadrage proposé repose sur les quatre éléments qui ont été mis en exergue: le rôle primordial de l'expert, le besoin de travailler à partir d'un cadre interprétatif, la nécessité d'envisager les niveaux micro- et macrostructurels, et l'importance de reconstituer les choix traductifs potentiels. La démarche de l'expert a été esquissée, accompagnée d'une mise en garde concernant les deux phénomènes particuliers que sont l'addition et la transformation. Il est toutefois clair qu'une telle démarche n'est jamais simple. C'est la raison pour laquelle je propose, dans les lignes qui suivent, d'examiner quelques exemples. 


\section{Créativité et absence de créativité dans des traductions de Pynchon et de Flaubert}

L'essentiel de mon analyse dans cette section portera sur deux traductions du deuxième roman du romancier américain Pynchon, dont le titre français est Vente à la criée du lot 49, et en croate Dražba predmeta 49. Cet ouvrage me semble particulièrement intéressant dans la mesure où, comme nous le verrons, il exige que son traducteur fasse preuve de créativité pour rendre quelques éléments clefs de l'œuvre. Dans un second temps, je donnerai un exemple de deux traductions anglaises de Madame Bovary, afin d'illustrer une démarche traductive pour le moins inattendue, et la difficulté de se prononcer sur son éventuelle créativité.

\subsection{Pynchon}

Le deuxième roman de Pynchon, The Crying of Lot 49, met en scène un conflit qui, selon le résumé qu'en fait Valtat,

est schématiquement un conflit d'interprétations situé à l'intérieur d'un même personnage, CEdipa Mass, devenue à sa grande surprise exécutrice testamentaire de son ancien amant, le magnat Inverarity Pierce ${ }^{16}$. Les possessions de Pierce, qui englobent tous les domaines, valent en quelque sorte pour un héritage de l'Amérique elle-même. En tentant de s'y retrouver, Edipa ne cesse de, ou pense sans cesse, se heurter à toutes sortes de signes témoignant de l'existence d'un réseau de communication occulte, réseau témoignant à son tour de l'existence d'une sorte de communauté définie par l'emploi de ce réseau, communauté elle-même occulte, donc, et qui constitue une sorte de double secret de l'Amérique, une Amérique invisible. Le conflit d'interprétation est entre, d'une part, l'existence réelle de ce réseau dont tout semble témoigner, et, d'autre part, la possibilité que ce réseau ne soit qu'un rêve né d'une lecture paranoïaque voulant lier à toutes forces des signes épars: conflit que le livre, en nous maintenant au bord de la révélation, ne résout pas. (Valtat 2006: 130)

L'héroïne, donc, mène une enquête dont l'objet premier est de démêler les affaires de Pierce Inverarity. Cependant, l'enquête devient rapidement une quête: Edipa se trouve devant la multiplication de signes manifestes dont elle ne parvient pas à trouver le sens, malgré ses nombreuses tentatives. Au centre du mystère se trouve (peut-être) ce réseau de communication occulte, une sorte de service postal secret, associé au nom de «Tristero». Les signes qu'elle découvre ont, probablement, un lien avec ce service: des timbres postaux (des faux...) ayant appartenu à Pierce, un graffiti représentant (apparemment) un cor postal muni d'une sourdine, et des motsacronymes qui ne livrent pas (entièrement) leur sens. L'accès ou le non-accès au(x) sens est, par conséquent, une thématique essentielle du roman; celle-ci fait naturellement partie du cadre interprétatif élaboré par l'expert, qui s'attend à ce que le lecteur de la traduction puisse accéder aux mêmes richesses d'interprétation. Pour le traducteur, il me semble que le seul moyen d'y parvenir est d'adopter une démarche créative.

J'ai choisi d'examiner un acronyme qui s'avère être essentiel dans le déroulement de l'intrigue, et dont on dénombre une vingtaine d'occurrences. Le terme "WASTE» est compris tout d'abord en tant que mot ordinaire signifiant déchet(s) ou gaspillage. Symbole ironique du système clandestin de distribution de courrier (il signifie à la fois le système lui-même et la boîte aux lettres où l'on doit poster le courrier), l'héroïne 
découvre relativement tard que le mot est aussi un acronyme, dont la signification est révélée sur un timbre postal.

(4) It turned out to be an old American stamp, bearing the device of the muted posthorn, belly-up badger, and the motto: WE AWAIT SILENT TRISTERO'S EMPIRE.

(Pynchon 1965/2012: 222 $2^{17}$ )

(a) Cela se révéla être un vieux timbre américain, marqué du cor postal à sourdine, avec comme devise WE AWAIT SILENT TRISTERO'S EMPIRE - Nous attendons l'empire de Tristero silencieux.

(Pynchon 1987: 195, traduction de Doury ${ }^{18}$ )

(b) Bila je to stara američka marka, sa znakom prigušenog poštanskog roga, jazavcem na leđima i natpisom: Očekujte Tristerov Pravedni i Apokaliptični Dolazak. [C'était un vieux timbre américain, avec le symbole du cor postal à sourdine, le blaireau sur le dos et la devise: attendez l'arrivée juste et apocalyptique de Tristero.]

(Pynchon 1998: 135, traduction de Šafran ${ }^{19}$ )

Tout au long de sa traduction, M. Doury garde la version anglaise de l'acronyme «WASTE». Il est, par conséquent, normal qu'au moment de cette révélation, il donne la signification des lettres en utilisant les mots anglais. Ne voulant pas laisser ses lecteurs dans l'ignorance, il intègre une traduction des mots dans le texte même, qui permet une compréhension au moins partielle de la problématique. Cependant, on est bel et bien dans un contexte de traduction, et le lecteur francophone qui n'a pas accès à l'anglais ne pourra pas comprendre le sens du mot qui constitue cet acronyme: cela fait tomber un pan du potentiel interprétatif du roman. Bien évidemment, on ne peut pas spéculer sur les raisons qui ont conduit à cette décision de non-traduction, ou de refus de créativité. Cependant, quand on reconstruit les choix traductifs potentiels, on voit qu'il aurait été possible de construire un acronyme français à partir de DÉCHET, par exemple «Discrets Et Calmes Hâtons l'Empire de Tristero». Enfin, il est intéressant de constater que le traducteur a choisi d'omettre de sa traduction l'expression «belly-up badger» qui, elle aussi, est un signe que l'héroïne s'efforce de déchiffrer. Le lecteur de la traduction ne peut que lire ce qui est sur la page: il ne peut en aucun cas soupçonner que la traduction qu'il lit est une version appauvrie.

Passons maintenant à la traduction de J. Šafran. La traductrice croate a souhaité donner à ses lecteurs la possibilité de comprendre les deux sens du mot «WASTE». Toutes les occurrences du terme sont traduites par le mot «OTPAD», qui signifie déchet. La phrase révélatrice, telle qu'elle est traduite en croate, correspond en français à: "C'était un vieux timbre américain, muni du signe du cor postal, le blaireau les quatre pattes en l'air, et la devise: Tenez-vous prêts pour l'arrivée légitime et apocalyptique de Tristero ${ }^{20 .}$ »Cette traduction est, sans aucun doute, conforme au cadre interprétatif. Elle est le résultat d'un travail conscient, motivé certainement par l'absence de solutions prévisibles ou existantes, et qui a exigé une expérimentation à partir de l'exigence de base, celle de garder à tout prix un acronyme possédant le même sens en tant que mot simple. Il convient de noter que la traductrice a également travaillé sur les autres acronymes (ce que le traducteur français n'a pas fait), en trouvant, par exemple, pour l'acronyme "DEATH", la traduction croate de mort ( $\mathrm{SMRT} »)$, ainsi qu'une solution de phrase une fois le sens de l'acronyme révélée ${ }^{1}$.

Ces deux exemples montrent les avantages d'une démarche créative et les inconvénients d'une stratégie de non-traduction. 


\subsection{Flaubert}

Le passage que je souhaite commenter est l'un de ceux que j'ai examinés dans mon ouvrage sur la critique des traductions (2011: 61). Il se trouve au début de la deuxième partie de Madame Bovary, lorsque les jeunes époux découvrent leur nouvelle demeure à Yonville. Comme je le note dans An Approach to Translation Criticism (2011: 36-41), l'un des éléments que j'ai retenus pour le cadre interprétatif concerne l'utilisation particulière de la syntaxe favorisée par Flaubert, qui lui permet de produire un effet de retardement et de mise en valeur dans de nombreuses descriptions, comme dans l'exemple qui suit.

(5) Emma, dès le vestibule, sentit tomber sur ses épaules, comme un linge humide, le froid du plâtre.

(Flaubert 1857/1971: 58 ${ }^{22}$ )

(a) The moment she stepped inside the entrance hall Emma felt the chill from the plaster walls fall on her shoulders, like the touch of a damp cloth.

(Flaubert 1957/1992: 108, traduction de Steegmuller ${ }^{23}$ )

(b) Emma, even in the hall, felt on her shoulders, like damp linen, the descending chill of the plaster.

(Flaubert 1992: 67, traduction de Wall ${ }^{24}$ )

La phrase de Flaubert est remarquable à plusieurs égards. En plaçant «Emma» en première position, le narrateur nous rend sensibles aux premières impressions de l'héroïne lorsqu'elle pénètre dans la maison. La proposition suivante, "dès le vestibule», juxtaposée au nom propre et antéposée avant le verbe, prépare le terrain en jouant sur l'implicite; une attente est ainsi créée: le lecteur attend des explications. C'est le début de l'effet de retardement: vient d'abord la sensation physique ("ses épaules»), puis le comparant («comme un linge humide») et, enfin, postposé et portant tout le poids de la fin de la phrase, le comparé - «le froid du plâtre» - ressenti également comme le symbole de sa vie avec Charles, et mis en exergue par la soirée qu'Emma vient de passer en compagnie de Léon à l'auberge du Lion d'or. La succession de virgules imprime un rythme entrecoupé à l'ensemble de la phrase qui, lui aussi, accentue la «révélation» concernant le froid du plâtre.

La phrase de Flaubert est également remarquable dans une optique de traduction. Il ne fait aucun doute que le traducteur anglophone est sensible à la présence d'une forte contrainte, qui le "pousse» à étoffer la proposition commençant par «dès», et à rétablir un ordre syntaxique plus prévisible - c'est-à-dire en insérant «le froid du plâtre» après le verbe principal. C'est ce qu'a fait Steegmuller: son étoffement consiste à introduire un verbe ("stepped») dans la proposition désormais antéposée; la syntaxe est remaniée dans une proposition également étoffée ("the chill from the plaster walls»), avec, comme bouquet final, un étoffement de la comparaison (ajout de «touch»). À quelques détails près, par conséquent, nous nous trouvons en présence d'une traduction prévisible, où les remaniements relèvent des réflexes semi-automatiques du traducteur expérimenté.

Wall a choisi de ne pas céder aux tentations d'une traduction plus ou moins standard $^{25}$. Il maintient la proposition avec dès en deuxième position dans la phrase, en optant pour une autre forme d'implicite (" $\underline{\text { even } ") ~ q u i, ~ c e r t e s, ~ n e ~ " d i t » ~ p a s ~ e x a c-~}$ tement la même chose que la proposition de Flaubert (on peut comprendre que 
l'héroïne s'étonne de ressentir cette sensation dans le vestibule). Encore plus remarquable est le refus de normaliser la syntaxe, mais, là aussi, il y a un prix à payer: «tomber» est transformé en gérondif et attaché à "chill», produisant, une nouvelle fois, un effet sensiblement différent (le choc est atténué). Sur le plan du sens, le résultat est mitigé, mais, sur le plan du style et dans le cadre d'une interprétation macrostructurelle, il est légitime d'estimer que nous sommes en présence d'un exemple de démarche créative qui permet d'entendre une voix narrative peu éloignée de celle du narrateur de Flaubert.

Nous pouvons tirer deux enseignements de cet exemple. Le premier concerne le jugement porté sur la traduction: il serait illusoire de vouloir trouver dans une traduction des choix qui remplissent tous les critères retenus à tous les niveaux. Il faut, je pense, saluer le travail du traducteur, sachant que toute démarche créative comporte des risques que le traducteur doit assumer. Le second enseignement concerne la démarche elle-même. Il me semble hautement probable que le traducteur ait passé du temps sur cette phrase, et que la solution trouvée résulte d'une insatisfaction à l'égard des traductions standard. La parenté évidente entre passages source et cible confirme la mise en garde que j'ai formulée ci-dessus concernant une analyse qui ne porterait que sur des traductions qui incarnent visiblement des remaniements de tous types.

\subsection{Bilan des exemples}

Il faut voir dans les quelques exemples choisis un tout petit échantillon des analyses que l'on peut mener afin d'arriver à une conclusion concernant la présence ou l'absence de créativité dans une traduction littéraire. On ne peut pas, sur la base de ces quelques passages, porter un jugement global sur le travail des traducteurs cités. On ne peut qu'admirer les exemples de créativité et regretter ceux qui, pour des raisons qui nous échappent, n’optent pas pour une démarche de ce type. Un grand chantier s'ouvre pour celui ou celle qui souhaite mener une analyse exhaustive d'une ouvre et de sa traduction.

\section{Conclusion}

Au terme de cette réflexion, je souhaite revisiter les paradoxes que j'ai mis en exergue dans mon introduction. J'en avais identifié quatre, que je vais résumer sous forme de simples oppositions: concevable versus inconcevable; visible versus invisible; désirable versus indésirable; global versus particulier. Il s'agit, en réalité, de quatre angles différents qui, pris ensemble, permettent une vision plus complète de la créativité. Inconcevable décrit le point de vue du grand public, tempéré par la réalité du phénomène de la créativité, forcément concevable. Invisible traduit l'expérience du lecteur du texte cible, opposée à la vision que peut avoir l'expert. Le couple désirableindésirable exprime le souhait et la crainte du traductologue. Enfin, particulier décrit la réalité propre à la créativité en traduction littéraire, par rapport à la vision usuelle d'une créativité globale. J'ai laissé entendre que ces paradoxes sont révélateurs, parce qu'ils permettent de comprendre la nature profonde de la créativité. C'est celle-ci, cette nature profonde, que je vais essayer de dégager en guise de conclusion, en proposant cinq caractéristiques fondamentales de cette compétence du traducteur 
littéraire. Le paradoxe désirable-indésirable met en lumière la prise de risque inhérente à la créativité, car la limite entre le désirable et l'indésirable est rapidement franchie. Les paradoxes visible-invisible et concevable-inconcevable illustrent la nature inattendue de la créativité. L’opposition entre global et particulier, où il a été question de "moments» de créativité (qui, bien entendu, peuvent ne pas exister), met en exergue la nature éphémère de la créativité. À ces caractéristiques, il convient d'en ajouter encore deux. La première est intimement liée à la nature, changeante, du texte source, qui a pour effet de rendre toute manifestation de créativité imprévisible. La deuxième découle de l'intervention de l'expert et du jugement qu'il formule: la créativité est nécessairement fragile. Il me semble que, par rapport aux critères que j'ai résumés dans la section 2 (ci-dessus), ces cinq caractéristiques donnent une image plus complète de ce phénomène très particulier. On peut espérer que l'apprenant, ou le traducteur littéraire en début de carrière, pourra s'en inspirer pour travailler plus efficacement cette compétence hautement souhaitable mais marquée par le sceau de sa propre fragilité.

\section{REMERCIEMENTS}

Je souhaite remercier Mathilde Fontanet et les experts anonymes de leur relecture attentive de cet article ainsi que de leurs propositions d'amélioration. J'adresse aussi mes remerciements à Jonathan Wilkinson qui, dans une communication personnelle, a longuement analysé l'exemple 5 (b).

\section{NOTES}

* Faculté de traduction et d'interprétation.

1. Voir le début de l’article que M. Ballard (1997: 85) consacre à la créativité: «Il peut paraître étrange, voire même provoquant, de parler de créativité à propos de la traduction quand on sait la mauvaise réputation que lui ont faite certains auteurs comme Du Bellay [...] et dont l'expression la plus cinglante se trouve sans doute chez Montesquieu où un traducteur ayant déclaré: "il y a vingt ans que je m’occupe à faire des traductions", se voit répondre: "Quoi! Monsieur... il y a vingt ans que vous ne pensez pas? Vous parlez pour les autres et ils pensent pour vous?" [...].»

2. Trésor de la langue française informatisé. Consulté le 21 juillet 2016, atilf.atilf.fr/.

3. Voir la définition de novel proposée par l'Oxford English Dictionary: «Of a new kind or nature; not previously imagined or thought of; (now) esp. interestingly new or unusual.» Consulté le 21 juillet 2016, oed.com/.

4. Tiberghien, Guy et ABDi, Hervé, dir. (2002): Dictionnaire des sciences cognitives. Paris: Armand Colin.

5. Le reproche que l'on peut adresser à Kussmaul concerne l'absence de critères concernant la nature d'une «bonne» traduction. À vrai dire, il n'existe aucun consensus en la matière. Certains traductologues se contentent de donner des indications très générales. Ladmiral, par exemple, propose le critère de la double fidélité ( fidèle à l’Esprit du texte (To) et conforme aux ressources spécifiques de la langue cible (Lt)» (2005: 482), tandis que Robinson (1991: 136) souligne qu'une traduction «is never "good" or "bad," never a success or failure in essence; it is pronounced good or bad by various people, and their pronouncements may vary in time and space».

6. La littérature comparée, par exemple, s'intéresse de près à la traduction, sans forcément faire référence aux travaux des traductologues.

7. Flaubert, Gustave (1886/2006): Madame Bovary. (Traduit par Eleanor MarX-Aveling) Munich: BookRix GmbH \& Co. KG. iBooks. https://itun.es/ch/_A-VZ.1. Mon article de 2014 s'intéresse à la traduction de la dimension culturelle du texte littéraire et n’aborde pas la créativité proprement dite. L'analyse du passage de Flaubert m'a permis de conclure que le choix de la traductrice «produit [...] un mini-effet de lecture, mais [...] ne dérange pas les grands équilibres culturels de cette partie du roman» (2014: 27). Il me semble raisonnable, par conséquent, de voir dans ce choix une instance de traduction créative. 
8. Gadamer (1996: 488-489) déclare: «Il est littéralement plus juste de dire que la langue nous parle que de dire que nous la parlons.»

9. Source: Oxford English Dictionary. Consulté le 21 juillet 2016, oed.com/.

10. Pinter, Harold (1975): No Man's Land. Londres: Eyre Methuen.

11. Pinter, Harold (1979): No Man's Land. (Adapté par Éric Kahane) Paris: Gallimard.

12. Simenon, Georges (1955/2012): Maigret et le corps sans tête. Paris: Omnibus (édition électronique).

13. Simenon, Georges (1967/1971): Maigret and the Headless Corpse. (Traduit par Ellen Ellenbogen) Fourth Omnibus. Harmondsworth: Penguin, 169-205.

14. Austen, Jane (1816/1966/1970): Emma. Harmondsworth: Penguin.

15. Austen, Jane (1996): Emma. (Traduit par Pierre Nordon) Paris: Livre de Poche.

16. J.-C. Valtat écrit le nom de famille, Inverarity, suivi du prénom, Pierce. Or, dans le roman, Pynchon écrit «Pierce Inverarity». Sachant que la plupart des noms propres semblent avoir un «vrai » sens, il importe de le lire dans son «sens» d'origine, où on peut comprendre l'idée de «percer» dans la «vérité», assortie d'un bruit (noise) supplémentaire («Inverarity»). Je dois cette observation au regretté Claude Richard.

17. Pynchon, Thomas (1965/2012): The Crying of Lot 49. New York: Penguin Press (édition électronique).

18. Pynchon, Thomas (1987): Vente à la criée du lot 49. (Traduit par Michel Doury) Paris: Seuil.

19. Pynchon, Thomas (1998): Dražba predmeta 49. (Traduit par Jasenka ŠAfran) Zagreb: Ceres.

20. Traduction de l'auteur.

21. L'acronyme en anglais: «Don't Ever Antagonize The Horn» (Pynchon (1965/2012: 158); en croate: «Suptrotstavljenje (sic) Moći Roga Traži» (Pynchon 1998: 96). Il y a une faute de frappe dans le texte en croate: on aurait dû lire «Suprotstavljenje».

22. Flaubert, Gustave (1857/1971): Madame Bovary. Paris: Garnier Frères.

23. Flaubert, Gustave (1957/1992): Madame Bovary. (Traduit par Francis Steegmuller) New York: Random House.

24. Flaubert, Gustave (1992): Madame Bovary. (Traduit par Geoffrey Wall) Harmondsworth: Penguin.

25. La traduction de Marx-Aveling citée ci-dessus suit la même logique que celle de Steegmuller. Les autres traductions étudiées dans Hewson 2011 suivent elles aussi cette logique de restructuration.

\section{RÉFÉRENCES}

Balacescu, Ioana et Stefanink, Bernd (2003a) : Modèles explicatifs de la créativité en traduction. Meta. 48(4):509-525.

Balacescu, Ioana et Stefanink, Bernd (2003b) : Du structuralisme au cognitivisme: la créativité au fil des théories de la traduction. Le langage et l'homme. 38(1):125-143.

BAlACESCu, Ioana et Stefanink, Bernd (2005) : Défense et illustration de l'approche herméneutique en traduction. Meta. 50(2):634-642.

BAllard, Michel (1997): Créativité et traduction. Target. 9(1):85-110.

Bastin, Georges L. (2003): Aventures et mésaventures de la créativité chez les débutants. Meta. 48(3):347-360.

Bayer-Hohenwarter, Gerrit (2011): “Creative Shifts” as a Means of Measuring and Promoting Translational Creativity. Meta. 56(3):663-692.

Berman, Antoine (1999): La traduction et la lettre ou l'Auberge du lointain. Paris: Seuil.

Dancette, Jeanne, Audet, Louise et Jay-Rayon, Laurence (2007): Axes et critères de la créativité en traduction. Meta. 52(1):108-122.

Delesse, Catherine et Richet, Bertrand (2009): Le coq gaulois à l'heure anglaise: analyse de la traduction anglaise d'Astérix. Arras: Artois Presses Université.

Englund Dimitrova, Birgitta (2005): Expertise and Explicitation in the Translation Process. Amsterdam/Philadelphie: John Benjamins Publishing Company.

Florin, Sider (1993) : Realia in translation. In: Palma Zlateva, dir. Translation as Social Action. Londres/New York: Routledge, 122-128.

Fontanet, Mathilde (2005): Temps de créativité en traduction. Meta. 50(2):432-447.

GADAMER, Hans-Georg (1996): Vérité et méthode: les grandes lignes d’une herméneutique philosophique. Paris: Éditions du Seuil. 
Guillemin-Flescher, Jacqueline (1986): Le linguiste devant la traduction. In: Fabienne Durand-Bogaert, dir. Traduire. Fabula 7. Paris: Presses universitaires de Lille, 59-68.

Hewson, Lance (2004): L’adaptation larvée: trois cas de figure. Palimpsestes. 16:105-116.

Hewson, Lance (2006): The Vexed Question of Creativity in Translation. Palimpsestes. Horssérie:53-63.

Hewson, Lance (2011): An Approach to Translation Criticism. Emma and Madame Bovary in translation. Amsterdam/Philadelphie: John Benjamins Publishing Company.

Hewson, Lance (2012): Traduire: les limites de la créativité. In: Annick Ettlin et Fabien Pillet, dir. Les Mouvements de la traduction. Genève: MétisPresses, 113-128.

Hewson, Lance (2014) : Baba, bouillie, brouet: les dangers de l'hybridité. Atelier de traduction. $22: 23-34$.

Hewson, Lance (2016): Les incertitudes du traduire. Meta. 61(1):12-28.

Hewson, Lance (2017): L'implicite: esquisse d'une approche traductologique. In: Sophie Anquetil, Juliette Elie-Deschamps et Cindy Lefebvre-Scodeller, dir. Autour des formes implicites. Rennes: Presses universitaires de Rennes, 209-230.

Koster, Cees (2000): From World to World. An Armamentarium For The Study Of Poetic Discourse In Translation. Amsterdam/Atlanta: Rodopi.

Kussmaul, Paul (1991): Creativity in the Translation Process: Empirical Approaches. In: Kitty M. van Leuven-Zwart et Ton Naaijkens, dir. Translation Studies: The State of the Art. Amsterdam/Atlanta: Rodopi, 91-101.

Kussmaul, Paul (2000a): Kreatives Übersetzen. Tübingen: Stauffenburg Verlag.

Kussmaul, Paul (2000b): Types of creative translating. In: Andrew Chesterman, Natividad Gallardo San Salvador et Yves Gambier, dir. Translation in Context. Amsterdam/ Philadelphie: John Benjamins Publishing Company, 117-126.

Ladmiral, Jean-René (2005): Le «salto mortale de la déverbalisation». Meta. 50(2):473-487.

LevÝ, Jiří (1963/1983/2011): The Art of Translation. (Traduit par Patrick Conness) Amsterdam/ Philadelphie: John Benjamins Publishing Company.

Newmark, Peter (1988): A Textbook of Translation. Londres: Prentice Hall.

Robinson, Douglas (1991): The Translator's Turn. Baltimore/Londres: The Johns Hopkins University Press.

Valtat, Jean-Christophe (2006): Entropie du suranné, néguentropie du déchet: Ubik et The Crying of Lot 49. In: Valérie Hugotte et Jean-Christope Valtat, dir. Modernités du suranné. Clermont-Ferrand: Presses universitaires Blaise-Pascal, 123-135. 